\title{
Are preoperative complete blood count parameters in peripheral nerve sheath tumors useful diagnostic tools?
}

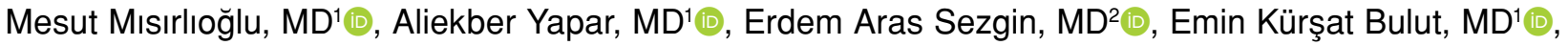

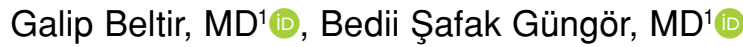

${ }^{1}$ Department of Orthopedics and Traumatology, Ankara Oncology Training and Research Hospital, Ankara, Turkey

Department of Orthopedics and Traumatology, Aksaray Training and Research Hospital, Aksaray, Turkey

Peripheral nerve sheath tumors (PNSTs) are soft tissue neoplasms, originating from peripheral nerves or cells associated with the nerves sheath. They range from benign schwannomas, which are the most common, to neurofibromas and malignant PNSTs (MPNSTs) with highly malignant characteristics and relatively rare. ${ }^{[1]}$ Differential diagnosis is usually made based on physical examination, clinical findings, location, genotype, and radiological imaging findings. Although PNSTs are seen on anatomical courses of nerves and, despite possible neurological symptoms and comprehensive radiological findings, preoperative diagnosis can be challenging, particularly in borderline PNSTs and those with atypical localization. ${ }^{[2]}$ Achieving a better prognosis in MPNSTs and reducing perioperative complications (i.e., iatrogenic neurovascular injury,

Received: October 06, 2020

Accepted: February 23, 2021

Published online: June 11, 2021

Correspondence: Mesut Mısırlıoğlu, MD. Ankara Onkoloji Eğitim ve Araştırma Hastanesi, Ortopedi ve Travmatoloji Kliniği, 06200 Demetevler, Ankara, Türkiye.

E-mail: mesutmisirlioglu@gmail.com

Doi: $10.52312 /$ jdrs.2021.79284

Citation: Mısırlıoğlu M, Yapar A, Sezgin EA, Bulut EK, Beltir G, Güngör BŞ. Are preoperative complete blood count parameters in peripheral nerve sheath tumors useful diagnostic tools?. Jt Dis Relat Surg 2021;32(2):340-346.

(C2021 All right reserved by the Turkish Joint Diseases Foundation

This is an open access article under the terms of the Creative Commons Attribution-NonCommercial License, which permits use, distribution and reproduction in any medium, provided the original work is properly cited and is not used for commercial purposes (http://creativecommons.org/licenses/by-nc/4.0/).

\section{ABSTRACT}

Objectives: This study aims to evaluate the diagnostic value of complete blood count (CBC) parameters in patients with peripheral nerve sheath tumors (PNSTs).

Patients and methods: A total of 181 patients (83 males, 98 females; median age: 44 years; range, 15 to 83 years) who underwent surgical treatment for PNSTs in our tertiary oncology center between January 2010 and December 2019 were retrospectively analyzed. Eighty-two patients were diagnosed with a neurofibroma, 79 with a schwannoma, and 20 with a malignant PNST (MPNST). The patient group was evaluated as malignant $(n=20)$ and benign $(n=161)$. Age- and sex-matched patients admitted to our outpatient clinic of orthopedic and traumatology with non-specific symptoms other than tumor, infection, fracture, and rheumatological or hematological diseases were included as the control group $(n=165)$. Data including age, sex, definitive histopathological diagnosis, and pre-treatment $\mathrm{CBC}$ values were obtained from the hospital records. Pre-treatment CBC values such as neutrophil-to-lymphocyte ratio (NLR), platelet-to-lymphocyte ratio (PLR), and lymphocyte-to-monocyte ratio (LMR) were calculated for both malignant and benign groups and control groups. Diagnostic values of NLR, PLR, and LMR between PNST groups were assessed using the receiver operating characteristic (ROC) curve analysis.

Results: Neurofibroma, schwannoma, and MNPST groups had significantly higher median NLR, compared to the control group $(\mathrm{p}<0.001)$, while the median LMR was significantly lower in these groups $(\mathrm{p}<0.05)$. However, the median PLR was higher only in the MPNST group, compared to the control group $(\mathrm{p}<0.001)$. Post-hoc analyses revealed that median NLR, PLR, and LMR ratios were similar in PNST groups, compared to the control group. In addition, the median NLR, PLR, and LMR ratios were similar between malignant and benign patient groups. The highest area under the curve (AUC) was found for NLR (AUC $=0.756)$ and LMR (AUC $=0.716)$ in the MPNST group.

Conclusion: Our study results suggest that NLR, PLR, and LMR may have an added value in the early diagnosis of PNSTs and are valuable for differentiating patients from healthy individuals, although their value in differential diagnosis is still unclear.

Keywords: Lymphocyte-to-monocyte ratio, malignant peripheral nerve sheath tumor, neurofibroma, neutrophil-to-lymphocyte ratio, platelet-to-lymphocyte ratio, schwannoma. 
inadequate resection, and motor and sensory loss) in benign PNSTs, relies on early diagnosis. ${ }^{[3]}$ As well as clinical and typical radiological findings, bioactive molecules, growth factors, proangiogenic factors, enzymes and cancer-related host inflammation factors are valuable in the diagnosis of these tumors. ${ }^{[4,5]}$ Despite ongoing researches, there is still no reliable, easily accessible, and clinically available biomarker. ${ }^{[6]}$ On the other hand, inflammatory biomarkers are increasingly being regarded as risk factors for tumor development, adhesion, angiogenesis and metastasis, although their exact mechanism of actions are still not well defined. ${ }^{[7,8]}$ Although it is not commonly used in the diagnosis of PNST preoperatively, many studies have been conducted with biomarkers such as S-100 protein, $\mathrm{CD}$ protein, growth-related protein, neurofibromin, and mitotic count values in tissue samples for histopathological diagnosis. ${ }^{[2,8]}$ Biomarkers which can be easily available preoperatively, such as complete blood count (CBC) parameters, have not been previously studied in the diagnosis of PNST.

The CBC parameters and relevant ratios derived such as neutrophil-to-lymphocyte ratio (NLR), platelet-to-lymphocyte ratio (PLR), and lymphocyteto-monocyte ratio (LMR) have been shown to be altered by systemic inflammatory response and are not only being used comprehensively in musculoskeletal tumors, but also in the diagnosis and evaluation of prognosis in many other tumors. ${ }^{[9-12]}$ Although platelets and neutrophils can promote growth, adhesion, and seeding of tumor, lymphocytes are the basic components of the adaptive and innate immune system and, thus, their decrease impairs the immune defense. ${ }^{[7,10]}$ Thrombocytosis, neutrophilia, and relatively low lymphocyte levels are also described in many tumoral tissues. ${ }^{[5]}$

In the light of these contemporary data, we hypothesized that aforementioned CBC parameters would have a previously unknown role as diagnostic tools in PNSTs which could help to reduce the diagnostic challenges. In the present study, therefore, we aimed to evaluate the diagnostic value of preoperative NLR, PLR, and LMR in the early and differential diagnosis of neurofibromas, schwannomas, and MPNSTs.

\section{PATIENTS AND METHODS}

This single-center, retrospective study was conducted at Ankara Oncology Training and Research Hospital between January 2010 and December 2019. Patients with a pathological diagnosis of PNST who received no oncological treatment, had primary surgical treatment, and had complete hospital records were included. Accordingly, 194 patients were identified through archive screening and 13 patients who did not meet inclusion criteria were excluded from the study. Exclusion criteria were as follows: any infectious disease and elevated white blood count (WBC) cells and C-reactive protein results, rheumatological, hematological, or inflammatory diseases, and the use of anti-inflammatory drugs. Finally, a total of 181 patients (83 males, 98 females; median age: 44 years; range, 15 to 83 years) who underwent surgical treatment for PNSTs were included. The patients were divided into three groups according to their confirmed histopathological diagnosis as schwannomas $(n=79)$, neurofibromas $(n=82)$, and MPNSTs $(n=20)$. The patients were further evaluated as malignant $(n=20)$ and benign ( $\mathrm{n}=161)$. Age- and sex-matched patients admitted to our outpatient clinic of orthopedic and traumatology with non-specific symptoms other than tumor, infection, fracture, and rheumatological or hematological diseases were included as the control group $(n=165)$. Data including age, sex, definitive histopathological diagnosis, and pre-treatment CBC values were obtained from the hospital records. A written informed consent was obtained from each participant. The study protocol was approved by Ankara Oncology Training and Research Hospital Ethics Committee (No: 2020-09-790). The study was conducted in accordance with the principles of the Declaration of Helsinki.

The WBC, neutrophil, lymphocyte, basophil, and platelet counts and other CBC parameters were recorded separately and the NLR and PLR were calculated by dividing the absolute count of neutrophils and platelets to lymphocytes among themselves. The LMR was calculated by dividing the count of lymphocytes to monocyte count.

\section{Statistical analysis}

Statistical analysis was performed using the IBM SPSS version 22.0 (IBM Corp., Armonk, NY, USA). Continuous variables were expressed in median (min-max), while categorical variables were expressed in number and percentage. Analytic methods (Kolmogorov-Smirnov and Shapiro-Wilk's test) were used to evaluate normal distributions. Data between the groups for continuous variables were not fit to normal distribution. While comparison analyses between two groups were done using the Mann-Whitney $\mathrm{U}$ test, comparisons of categorical variables between independent groups were done using the chi-square test. Cut-off values for NLR, PLR, and 


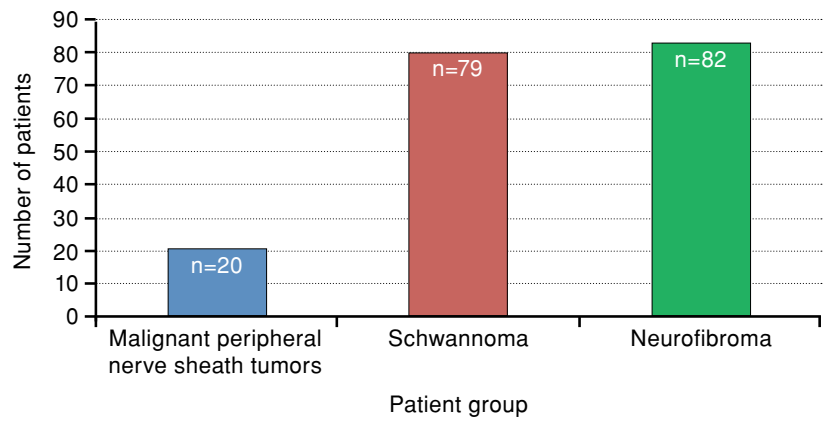

FIGURE 1. Distribution of patients according to diagnosis.

LMR defined by receiver operating characteristic (ROC) curve analysis were used to classify patients in the PNST and control groups. Additionally, the area under the curve (AUC), cut-off values, sensitivity and specificity of these cut-offs values, positive likelihood ratio (+LHR), positive predictive value (PPV) and negative predictive value (NPV) were calculated for each parameter and the results were compared. A $p$ value of $<0.05$ was considered statistically significant.

\section{RESULTS}

The median age was 43.5 (range, 15 to 83 ) years in the neurofibroma group, 43 (range, 15 to 76 ) years in the schwannoma group, 48.5 (range, 22 to 79) years in the MPNST group, and 43 (range, 15 to 76) in the control group (Figure 1). There was no statistically significant difference in the age and sex distribution among the groups (Table I).

While the median NLR was significantly higher in neurofibroma $(p<0.001)$, schwannoma $(p<0.001)$, and MPNST $(p<0.001)$ groups, compared to the control group, the median LMR was significantly lower in these groups $(\mathrm{p}=0.004, \mathrm{p}=0.005$, and $\mathrm{p}=0.002$, respectively). The MPNST group was the only group with a significantly higher median PLR, compared to the control group $(\mathrm{p}<0.001)$. No significant difference between the PNST groups was found for NLR, PLR, and LMR in post-hoc analyses ( $p>0.05)$. In addition, the median NLR ( $\mathrm{p}=0.430)$, PLR $(\mathrm{p}=0.152)$, and LMR $(p=0.286)$ values were similar in malignant and benign groups (Table I).

\begin{tabular}{|c|c|c|c|c|}
\hline \multicolumn{5}{|c|}{$\begin{array}{c}\text { TABLE I } \\
\text { raphic characteristics and CBC parameters } 0\end{array}$} \\
\hline & $\begin{array}{c}\text { Neurofibroma }\left(\mathrm{G}_{N}\right) \\
(\mathrm{n}=82)\end{array}$ & $\begin{array}{c}\text { Schwannoma (Gs) } \\
\qquad(n=79)\end{array}$ & $\begin{array}{l}\operatorname{MPNST}\left(\mathrm{G}_{M}\right) \\
(\mathrm{n}=20)\end{array}$ & $\begin{array}{c}\text { Control }\left(G_{c}\right) \\
(n=165)\end{array}$ \\
\hline Age (year)†‡ & $43.5(15-83)$ & $43(15-76)$ & 48.5 (22-79) & $43(15-76)$ \\
\hline$p$ values* & \multicolumn{2}{|c|}{$\mathrm{G}_{\mathrm{N}}$ vs. $\mathrm{G}_{\mathrm{C}}=0.414$} & \multicolumn{2}{|c|}{$G_{N} v s . G_{S}=0.765$} \\
\hline & \multicolumn{2}{|c|}{$\mathrm{G}_{\mathrm{S}}$ vs. $\mathrm{G}_{\mathrm{C}}=0.558$} & \multicolumn{2}{|c|}{$\mathrm{G}_{N}$ vs. $\mathrm{G}_{\mathrm{M}}=0.670$} \\
\hline & \multicolumn{2}{|c|}{$\mathrm{G}_{\mathrm{M}}$ vs. $\mathrm{G}_{\mathrm{C}}=0.237$} & \multicolumn{2}{|c|}{$\mathrm{G}_{M}$ vs. $\mathrm{G}_{S}=0.604$} \\
\hline \multicolumn{5}{|l|}{ Sex, n (\%) } \\
\hline Female & 47 (57.3) & $41(51.9)$ & $10(50)$ & 88 (53.3) \\
\hline Male & $35(47.2)$ & $38(48.1)$ & $10(50)$ & $77(46.7)$ \\
\hline \multirow[t]{3}{*}{$p$ values ${ }^{* *}$} & \multicolumn{2}{|c|}{$\mathrm{G}_{N}$ vs. $\mathrm{G}_{\mathrm{C}}=0.558$} & \multicolumn{2}{|c|}{$\mathrm{G}_{N}$ vs. $\mathrm{G}_{S}=0.496$} \\
\hline & \multicolumn{2}{|c|}{$\mathrm{G}_{s}$ vs. $\mathrm{G}_{\mathrm{c}}=0.834$} & \multicolumn{2}{|c|}{$\mathrm{G}_{N}$ vs. $\mathrm{G}_{\mathrm{M}}=0.565$} \\
\hline & \multicolumn{2}{|c|}{$\mathrm{G}_{M}$ vs. $\mathrm{G}_{C}=0.782$} & \multicolumn{2}{|c|}{$\mathrm{G}_{\mathrm{M}}$ vs. $\mathrm{G}_{S}=0.882$} \\
\hline NLR†‡ & $2.29(0.65-13.7)$ & $2.38(0.7-24.2)$ & $2.41(1.36-9.1)$ & $1.85(0.11-9.18)$ \\
\hline \multirow[t]{3}{*}{$p$ values* } & \multicolumn{2}{|c|}{$G_{N}$ vs. $G_{C}<0.001$} & \multicolumn{2}{|c|}{$G_{N} v s . G_{S}=0.956$} \\
\hline & \multicolumn{2}{|c|}{$\mathrm{G}_{s}$ vs. $\mathrm{G}_{\mathrm{c}}<0.001$} & \multicolumn{2}{|c|}{$\mathrm{G}_{N}$ vs. $\mathrm{G}_{\mathrm{M}}=0.456$} \\
\hline & \multicolumn{2}{|c|}{$\mathrm{G}_{M}$ vs. $\mathrm{G}_{\mathrm{C}}<0.001$} & \multicolumn{2}{|c|}{$\mathrm{G}_{\mathrm{M}}$ vs. $\mathrm{G}_{\mathrm{S}}=0.454$} \\
\hline PLR†‡ & $141.1(58.9-570.8)$ & $131.6(66.7-774.6)$ & $156.3(62.7-409.8)$ & $129.7(63.7-296.7)$ \\
\hline \multirow[t]{3}{*}{$p$ values ${ }^{*}$} & \multicolumn{2}{|c|}{$\mathrm{G}_{N}$ vs. $\mathrm{G}_{\mathrm{C}}=0.120$} & \multicolumn{2}{|c|}{$G_{N}$ vs. $G_{S}=0.606$} \\
\hline & \multicolumn{2}{|c|}{$\mathrm{G}_{\mathrm{S}}$ vs. $\mathrm{G}_{\mathrm{C}}=0.375$} & \multicolumn{2}{|c|}{$\mathrm{G}_{N}$ vs. $\mathrm{G}_{\mathrm{M}}=0.211$} \\
\hline & \multicolumn{2}{|c|}{$\mathrm{G}_{\mathrm{M}}$ vs. $\mathrm{G}_{\mathrm{C}}=0.027$} & \multicolumn{2}{|c|}{$\mathrm{G}_{\mathrm{M}}$ vs. $\mathrm{G}_{S}=0.143$} \\
\hline LMR†‡ & $4.25(0.82-9.18)$ & $4.12(1.03-12.38)$ & $3.71(0.82-7.79)$ & $4.81(1.1-9.13)$ \\
\hline \multirow[t]{3}{*}{$p$ values* } & \multicolumn{2}{|c|}{$\mathrm{G}_{N}$ vs. $\mathrm{G}_{\mathrm{C}}=0.004$} & \multicolumn{2}{|c|}{$\mathrm{G}_{N}$ vs. $\mathrm{G}_{S}=0.723$} \\
\hline & \multicolumn{2}{|c|}{$\mathrm{G}_{S}$ vs. $\mathrm{G}_{\mathrm{C}}=0.005$} & \multicolumn{2}{|c|}{$\mathrm{G}_{N}$ vs. $\mathrm{G}_{\mathrm{M}}=0.365$} \\
\hline & \multicolumn{2}{|c|}{$\mathrm{G}_{M}$ vs. $\mathrm{G}_{C}=0.002$} & $\mathrm{G}_{M} v s$ & $=0.263$ \\
\hline
\end{tabular}


(a)

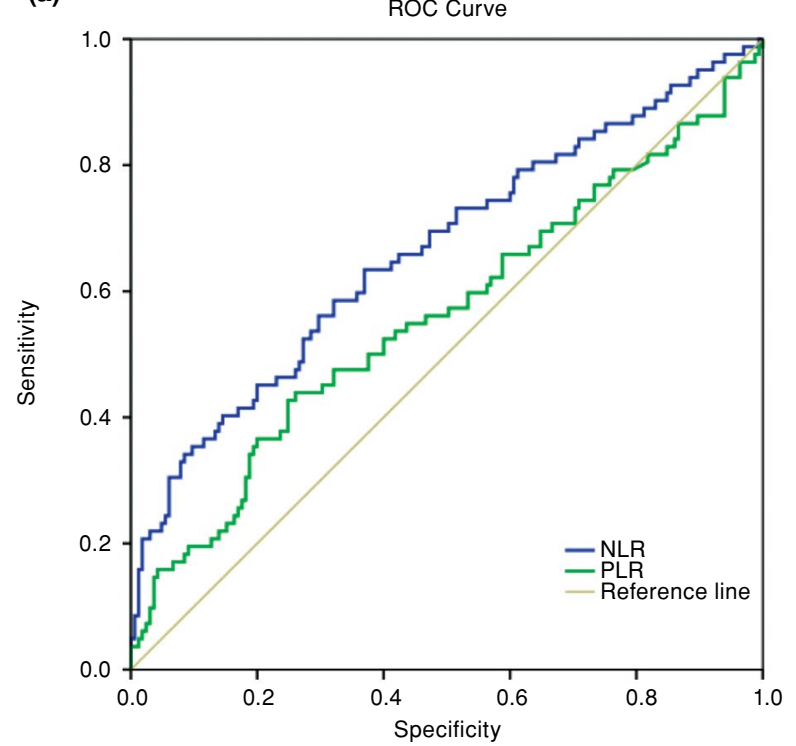

(b)

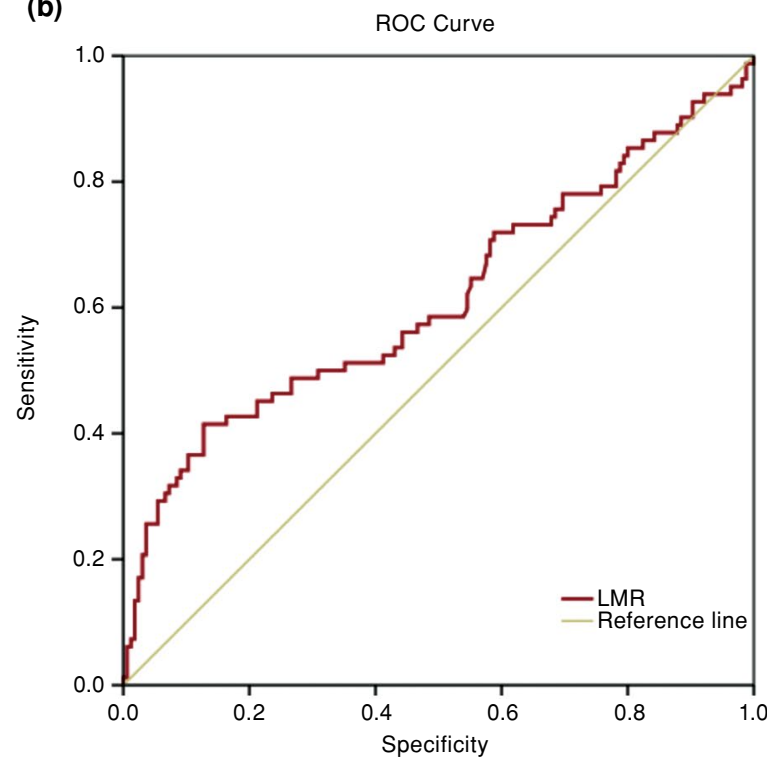

FIGURE 2. Receiver operating characteristic curves for NLR, PLR, and LMR.

(a) Larger results of NLR and PLR indicate a more diagnostic positive test for neurofibromas. (b) Smaller results of LMR indicate a more diagnostic positive test for neurofibromas.

NLR: Neutrophil-to-lymphocyte ratio; PLR: Platelet-to-lymphocyte ratio; LMR: Lymphocyte-to-monocyte ratio.

The presence of statistically significant cut-off values and diagnostic predictability of NLR, PLR, and LMR were evaluated with the ROC analysis (Figures 2-4). The highest AUC was obtained in the MPNST group for NLR $(\mathrm{AUC}=0.756)$ and

(a)

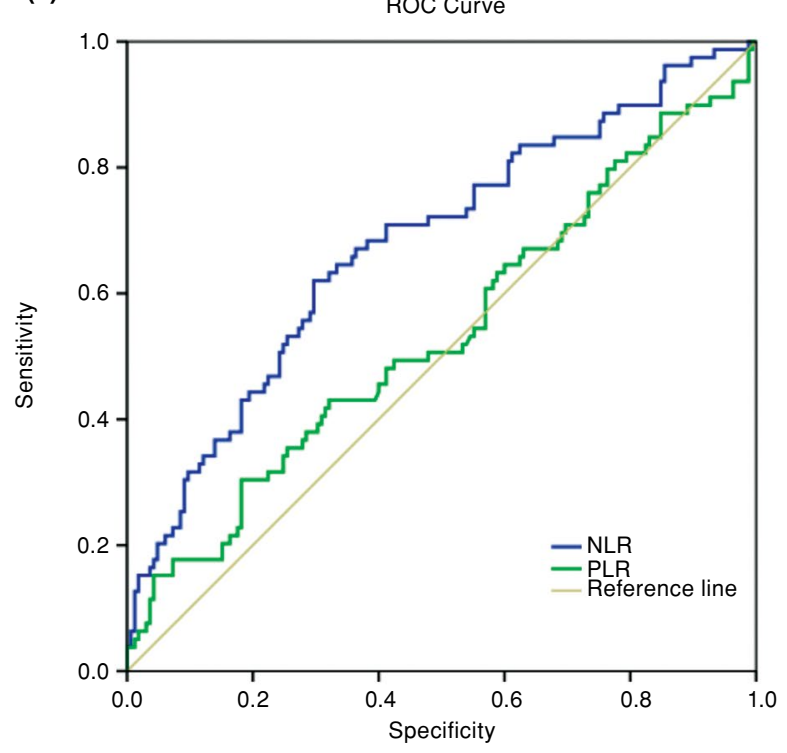

LMR (AUC=0.716) (Table II). While the AUC for other groups were also statistically significant, they showed a low predictability. The cut-off values for ratios, sensitivity, specificity, +LHR, PPV, and NPV values are presented in Table II.

(b)

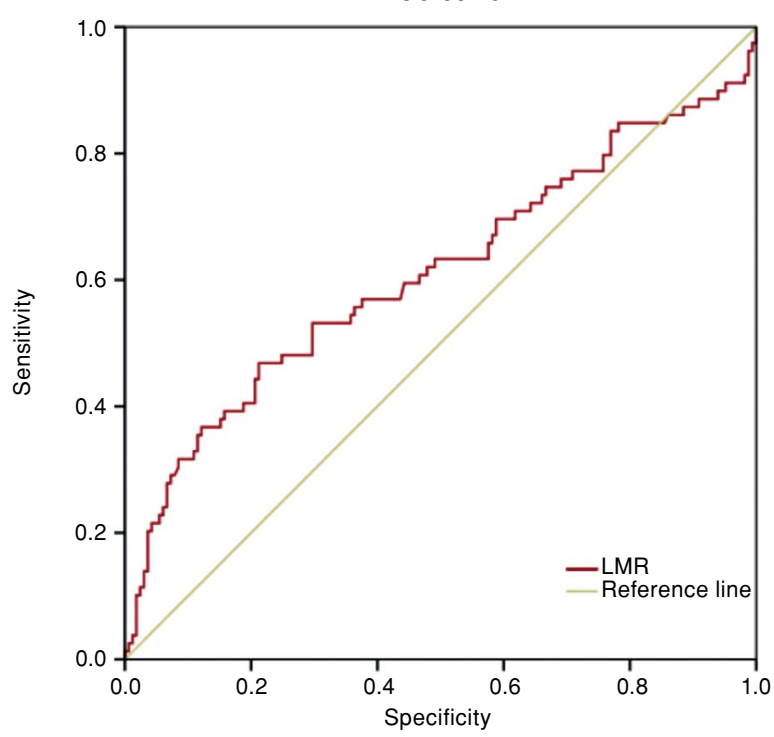

FIGURE 3. Receiver operating characteristic curves for NLR, PLR, and LMR

(a) Larger results of NLR and PLR indicate a more diagnostic positive test for schwannomas. (b) Smaller results of LMR indicate a more diagnostic positive test for schwannomas.

NLR: Neutrophil-to-lymphocyte ratio; PLR: Platelet-to-lymphocyte ratio; LMR: Lymphocyte-to-monocyte ratio. 
(a)

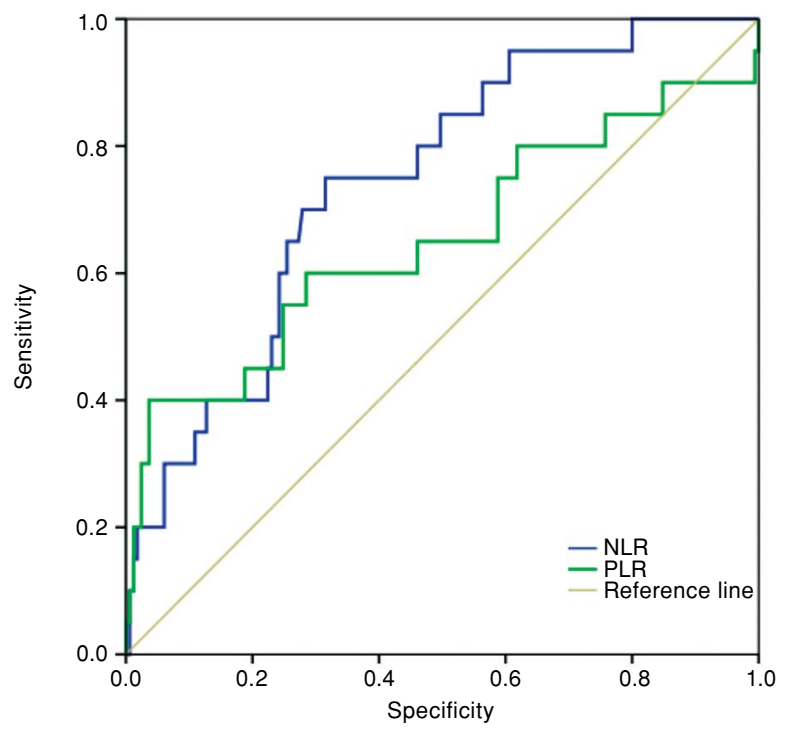

(b)

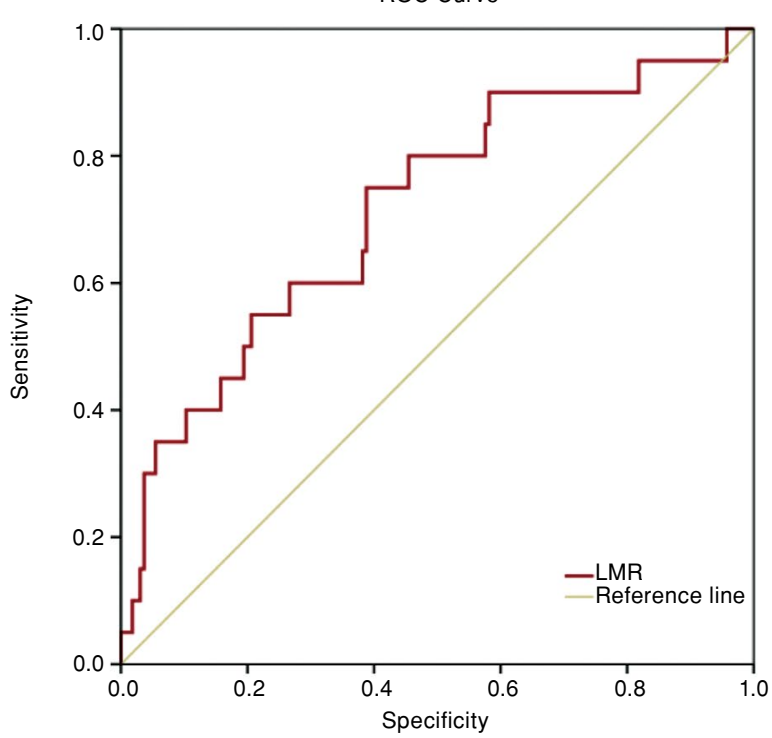

FIGURE 4. Receiver operating characteristic curves for NLR, PLR, and LMR.

(a) Larger results of NLR and PLR indicate a more diagnostic positive test for MPNSTs. (b) Smaller results of LMR indicate a more diagnostic positive test for MPNSTs.

MPNST: Malignant peripheral nerve sheath tumor; NLR: Neutrophil-to-lymphocyte ratio; PLR: Platelet-to-lymphocyte ratio; LMR: Lymphocyte-to-monocyte ratio.

\section{TABLE II}

Predictive values of preoperative NLR, LMR, and PLR ratios for MPNSTs, schwannomas, and neurofibromas in diagnosis ( $\mathrm{n}=337$ )

\begin{tabular}{lcccccccc} 
& AUC $(95 \% \mathrm{Cl})$ & $p$ & Cut-off & Sensitivity (\%) & Specificity (\%) & +LHR & PPV (\%) & NPV (\%) \\
\hline NLR & $0.664(0.590-0.739)$ & $<0.001$ & $\geq 2.03$ & 63.4 & 63 & 1.71 & 45.2 & 77.3 \\
PLR & $0.561(0.481-0.641)$ & 0.120 & $\geq 142.6$ & 50 & 62.4 & 1.33 & 39.8 & 71.5 \\
LMR & $0.614(0.533-0.694)$ & 0.004 & $\leq 4.19$ & 50 & 69.1 & 1.62 & 44.6 & 73.5 \\
NLR & $0.678(0.604-0.751)$ & $<0.001$ & $\geq 2.12$ & 64.6 & 66.7 & 1.93 & 48.1 & 79.7 \\
PLR & $0.535(0.455-0.615)$ & 0.375 & $\geq 136.6$ & 49.4 & 57.6 & 1.16 & 35.8 & 70.4 \\
LMR & $0.610(0.528-0.692)$ & 0.005 & $\leq 4.19$ & 53.2 & 69.1 & 1.72 & 45.2 & 75.5 \\
NLR & $0.744(0.639-0.850)$ & $<0.001$ & $\geq 2.27$ & 70 & 72.1 & 2.51 & 23 & 95.2 \\
PLR & $0.651(0.497-0.805)$ & 0.027 & $\geq 151$ & 60 & 71.5 & 2.1 & 20.3 & 93.7 \\
LMR & $0.716(0.591-0.841)$ & 0.002 & $\leq 4.46$ & 75 & 61.2 & 1.93 & 19.0 & 95.3
\end{tabular}

NLR: Neutrophil-to-lymphocyte ratio; LMR: Lymphocyte-to-monocyte ratio; PLR: Platelet-to-lymphocyte ratio; MPNST: Malignant peripheral nerve sheath tumor; AUC: Area under the curve; Cl: Confidence interval; +LHR: Positive likelihood ratio; PPV: Positive predictive value; NPV: Negative predictive value;

The statistically significant cut-off for NLR was $\geq 2.03$ (sensitivity $=63.4 \%$, specificity=63\%), $\leq 4.19$ (sensitivity $=50 \%$, specificity $=69.1 \%$ ) for LMR, and $\geq 142.6$ (sensitivity $=50 \%$, specificity $=62.4 \%$ ) for PLR, indicating that NLR, PLR, and LMR had a diagnostic value with statistically significant cut-off values.

\section{DISCUSSION}

In the current study, we evaluated the role of factors that portray systemic inflammatory response in diagnosis of PNST. Our results showed that pretreatment NLR, PLR, and LMR values obtained from peripheral blood had a diagnostic value in PNST patients, compared to healthy controls. In the literature, there are studies showing the diagnostic significance of pre-treatment $C B C$ parameters in many soft tissue tumors. ${ }^{[9-14]}$ In line with previous studies, our results demonstrated that CBC parameters were associated with the diagnosis of PNST. It seems plausible to perform in-depth analysis in a patient investigated for PNST, who has 
a high clinical suspicion, high NLR, PLR, and low LMR values. Also, when the PLR ratio is high, we should suspect more MPNST in the diagnosis of the nerve tumors.

Systemic inflammatory biomarkers such as NLR, PLR, and LMR have recently started to be used as alternatives to conventional methods in the diagnosis and management of different types of tumors. ${ }^{[5,9-17]}$ The use of CBC parameters in the clinical setting as screening tools in diagnosis and prognosis of musculoskeletal tumors has been gaining popularity due to the inexpensive, non-invasive, and being easy to access nature of this method. ${ }^{[17]}$ There are reports showing that the number of neutrophils that produce reactive oxygen species, which induce tumor growth, angiogenesis and metastasis, is increased more around malignant tumor cells. ${ }^{[7]}$ Neutrophilia, decreasing the lymphocyte-mediated immune response, can prohibit the immune system by T-cell suppression. Platelets recruited by the influence of tumor cells can protect them from the immune response, thereby, promoting their dissemination. ${ }^{[8,10]}$ Consistent with the literature, our study showed that PNST patients had elevated neutrophil and monocyte count with a decline in lymphocyte count.

There are many contemporary studies reporting the role of biomarkers derived from $\mathrm{CBC}$ in the differential diagnosis of tumors. In a study performed in 503 spinal tumors, NLR and PLR were found to be valuable markers in preoperative diagnosis and differentiation of primary and secondary tumors. ${ }^{[10]}$ Cut-off values for NLR $(>3.19)$ and PLR $(>141)$ were also reported to be associated with a high risk of malignancy. In our study, statistically significant optimal diagnostic cut-off values for NLR $(\geq 2.03)$, PLR $(\geq 142.6)$ and LMR $(\leq 4.19)$ had a diagnostic value for distinguishing PNSTs from non-PNSTs.

Ozyalvacli et al. ${ }^{[11]}$ showed that NLR was useful in differentiation of breast cancer and benign proliferative conditions of the breast, as well as in the diagnosis and prognosis of breast carcinomas. In another study in lung carcinomas, annual changes of NLR and PLR were found to have a diagnostic and developmental value, compared to healthy controls. ${ }^{[12]}$ On the other hand, canine studies showed that higher NLR with reduced albumin/globulin ratio might be considered the indicators of sarcomas during differentiation malignant tumors from benign ones. ${ }^{[13]}$ However, the authors concluded that these values had no role in grading of the sarcoma. Similarly, NLR was found to be a useful biomarker in the diagnosis and prognosis of extremity and trunk soft-tissue sarcomas in another study. ${ }^{[14]}$ It was also reported that NLR and LMR had a diagnostic value in cartilage tumors, such as enchondromas and low-grade chondrosarcomas. ${ }^{[15]}$ Although our results showed that NLR, PLR, and LMR were significantly correlated with the diagnosis of PNSTs, inflammatory variables could be supportive in diagnosing PNSTs such as lung and breast carcinomas, spinal and cartilaginous tumors. These results indicate that the $\mathrm{CBC}$ biomarker may have an added value in the early diagnosis of PNST.

It can be argued that, as well as aiding in diagnosis, these parameters can also influence the decision-making process of the treating physician. In a study investigating the prognosis and treatment outcomes of the musculoskeletal soft tissue sarcomas, NLR was found to be more valuable as a predictor than PLR and other CBC biomarkers. ${ }^{[16]}$ A meta-analysis including 40,559 solid malignant tumors also showed a median cut-off value of NLR ( $\geq 4)$ to be associated with poor prognosis. ${ }^{[17]}$ Despite these encouraging results, as there are many serum-based inflammatory diagnostic markers providing controversial results, we suggest that NLR, PLR, and LMR should be considered to be used in the diagnosis of PNSTs with the combination of conventional methods. Although our results do not support the use of preoperative NLR, PLR, and LMR to differentiate neurofibromas, schwannomas, and MPNSTs, compared to healthy population, they still have a diagnostic value in the management of PNSTs.

We acknowledge the limitations to our study first being its retrospective and single-center design. In addition, systemic inflammatory biomarkers inevitably had intrinsic weaknesses, as they were non-specific predictors of PNSTs. Another limitation is the limited sample size of MPNSTs due to their low incidence. Also, although the cut-off values were statistically significant, their sensitivity and specificity were low. The main strengths of our study are the utilization of $\mathrm{CBC}$ biomarkers, which are simple, inexpensive, and readily available test, and exclusion of patient groups which may affect CBC parameters.

In conclusion, pre-treatment NLR, PLR, and LMR appear to have a diagnostic value in the PNSTs, compared to healthy individuals; however, their diagnostic value in the differential diagnosis still remains to be elucidated. The NLR emerges as a more precise differentiating factor in the diagnosis of PNST than PLR and LMR. Although our results do not allow us to strongly recommend the use of NLR, PLR, and LMR as sole variables in the differential diagnosis and treatment planning of PNSTs, they 
should be considered combined with other diagnostic parameters and can be useful as a basis for future researches.

\section{Declaration of conflicting interests}

The authors declared no conflicts of interest with respect to the authorship and/or publication of this article.

\section{Funding}

The authors received no financial support for the research and/or authorship of this article.

\section{REFERENCES}

1. Rodriguez FJ, Folpe AL, Giannini C, Perry A. Pathology of peripheral nerve sheath tumors: Diagnostic overview and update on selected diagnostic problems. Acta Neuropathol 2012;123:295-319.

2. Meyer A. Review and update in the diagnosis of peripheral nerve sheath tumors. Curr Opin Neurol 2020;33:575-86.

3. Park SJ, Sawitzki B, Kluwe L, Mautner VF, Holtkamp N, Kurtz A. Serum biomarkers for neurofibromatosis type 1 and early detection of malignant peripheral nerve-sheath tumors. BMC Med 2013;11:109.

4. Mantovani A, Allavena P, Sica A, Balkwill F. Cancer-related inflammation. Nature 2008;454:436-44.

5. Paramanathan A, Saxena A, Morris DL. A systematic review and meta-analysis on the impact of pre-operative neutrophil lymphocyte ratio on long term outcomes after curative intent resection of solid tumours. Surg Oncol 2014;23:31-9.

6. Schaefer IM, Fletcher CDM. Recent advances in the diagnosis of soft tissue tumours. Pathology 2018;50:37-48.

7. Grivennikov SI, Greten FR, Karin M. Immunity, inflammation, and cancer. Cell 2010;140:883-99.

8. Diakos CI, Charles KA, McMillan DC, Clarke SJ. Cancerrelated inflammation and treatment effectiveness. Lancet Oncol 2014;15:e493-503.

9. Prodromidou A, Andreakos P, Kazakos C, Vlachos DE, Perrea D, Pergialiotis V. The diagnostic efficacy of platelet- to-lymphocyte ratio and neutrophil-to-lymphocyte ratio in ovarian cancer. Inflamm Res 2017;66:467-75.

10. Li Y, Wang B, Zhou S, Jiang L, Yang S, Liu X, et al. Do routine blood test results help in the diagnosis of spine tumors? A retrospective study of the significance of pretreatment neutrophil-to-lymphocyte and platelet-tolymphocyte ratios from 503 spine tumor patients. Medicine (Baltimore) 2019;98:e14902.

11. Ozyalvacli G, Yesil C, Kargi E, Kizildag B, Kilitci A, Yilmaz F. Diagnostic and prognostic importance of the neutrophil lymphocyte ratio in breast cancer. Asian Pac J Cancer Prev 2014;15:10363-6.

12. Sanchez-Salcedo P, de-Torres JP, Martinez-Urbistondo D, Gonzalez-Gutierrez J, Berto J, Campo A, et al. The neutrophil to lymphocyte and platelet to lymphocyte ratios as biomarkers for lung cancer development. Lung Cancer 2016;97:28-34.

13. Macfarlane L, Morris J, Pratschke K, Mellor D, Scase $\mathrm{T}$, Macfarlane $\mathrm{M}$, et al. Diagnostic value of neutrophillymphocyte and albumin-globulin ratios in canine soft tissue sarcoma. J Small Anim Pract 2016;57:135-41.

14. Idowu OK, Ding $Q$, Taktak AF, Chandrasekar CR, Yin Q. Clinical implication of pretreatment neutrophil to lymphocyte ratio in soft tissue sarcoma. Biomarkers 2012;17:539-44.

15. Yapar A, Ulucaköy C, Sezgin EA, Atalay İB, Ekşioğlu MF. Diagnostic role of neutrophil-to-lymphocyte ratio and monocyte-to-lymphocyte ratio in patients with enchondroma and low-grade chondrosarcoma. Jt Dis Relat Surg 2020;31:286-90.

16. Viñal D, Martinez D, Garcia-Cuesta JA, Gutierrez-Sainz L, Martinez-Recio S, Villamayor J, et al. Prognostic value of neutrophil-to-lymphocyte ratio and other inflammatory markers in patients with high-risk soft tissue sarcomas. Clin Transl Oncol 2020;22:1849-56.

17. Templeton AJ, McNamara MG, Šeruga B, Vera-Badillo FE, Aneja P, Ocaña A, et al. Prognostic role of neutrophil-tolymphocyte ratio in solid tumors: A systematic review and meta-analysis. J Natl Cancer Inst 2014;106:dju124. 